\title{
p53 overexpression in morphologically ambiguous endometrial carcinomas correlates with adverse clinical outcomes
}

\author{
Karuna Garg ${ }^{1}$, Mario M Leitao $\mathrm{Jr}^{2}$, Christine A Wynveen ${ }^{1}$, Gabriel L Sica ${ }^{1}$, Jinru Shia ${ }^{1}$, \\ Weiji Shi ${ }^{3}$ and Robert A Soslow ${ }^{*, 1}$
${ }^{1}$ Department of Pathology, Memorial Sloan Kettering Cancer Center, New York, NY, USA; ${ }^{2}$ Department of Surgery, Gynecology Service, Memorial Sloan Kettering Cancer Center, New York, NY, USA and ${ }^{3}$ Department \\ of Epidemiology and Biostatistics, Memorial Sloan Kettering Cancer Center, New York, NY, USA
}

\begin{abstract}
The distinction between uterine serous and endometrioid carcinomas can usually be achieved by morphologic examination alone. However, there are occasional 'morphologically ambiguous endometrial carcinomas' that show overlapping serous and endometrioid features and defy histologic classification. The primary aim of this study was to assess the clinical significance of p53 overexpression using immunohistochemistry in such tumors. Related aims included (1) assessing interobserver diagnostic concordance for histologic subclassification of these tumors using a panel of pathologists with and without gynecologic pathology expertise and (2) elucidating the histologic features that correlate with p53 status. Thirty-five such cases were identified during the study period. p53 overexpression was seen in 17 of 35 cases. Tumors with p53 overexpression were associated with a significantly inferior progression-free survival and disease-specific survival compared with those that lacked p53 overexpression (3-year progression-free survival and disease-specific survival were 94 and $100 \%$ in patients with no $\mathrm{p} 53$ overexpression, and 52 and $54 \%$ in patients with p53 overexpression; $P=0.02$ and 0.003 , respectively). The consensus diagnosis rendered by gynecologic pathologists was predictive of disease-specific survival $(P=0.002)$, but not progression-free survival $(P=0.11)$. Although the interobserver diagnostic concordance $(\mathrm{kappa}=\mathbf{0 . 7 0})$ was substantial for gynecologic pathologists, and highly associated with p53 status $(77 \%$ of 'favor serous' cases showed p53 overexpression, whereas only $25 \%$ of 'favor endometrioid' cases showed p53 overexpression; $P=0.005$ ), the concordance between the consensus diagnosis of the two specialized pathologists versus each of three non-specialized pathologists was poor $(k a p p a=0.13-0.25)$. The histologic feature that correlated most with p53 overexpression was the presence of diffuse high nuclear grade. p53 immunohistochemistry assays in morphologically ambiguous endometrial carcinomas are roughly as clinically informative as gynecologic pathology consultation and can be helpful for prognostic assessment and therapeutic decision making in difficult endometrial carcinomas.

Modern Pathology (2010) 23, 80-92; doi:10.1038/modpathol.2009.153; published online 23 October 2009
\end{abstract}

Keywords: p53; endometrial carcinoma; outcome

Endometrial carcinomas are a heterogeneous group of tumors with variable morphology and clinical behavior. Histologic subclassification of these tumors is important as it has significant therapeutic and prognostic implications. Although this can be

${ }^{*}$ Correspondence: Professor RA Soslow, MD, Department of Pathology, Memorial Sloan Kettering Cancer Center, 1275 York Avenue, MRI-1027, New York, NY 10065, USA.

E-mail: soslowr@mskcc.org

Received 19 July 2009; revised 28 August 2009; accepted 29 August 2009; published online 23 October 2009 achieved with examination of hematoxylin and eosin-stained (H\&E) slides alone in most cases, there are some morphologically ambiguous carcinomas that defy reproducible, accurate and clinically meaningful histologic classification. These tumors show overlapping morphologic features of endometrial endometrioid carcinomas and uterine serous carcinomas. $^{1,2}$

Endometrial carcinomas are divided into types 1 and 2 as described by Bokhman. ${ }^{3}$ Endometrioid carcinomas or type 1 tumors are typically low-grade tumors that are associated with estrogen excess and 
pursue favorable clinical outcomes, whereas serous carcinomas are prototypic type 2 tumors that are high-grade, aggressive tumors associated with poor clinical outcomes. Type 1 tumors are usually associated with microsatellite instability and mutations in PTEN, CTNB1 (beta-catenin), $k-R A S$ and PIK3CA. Type 2 endometrial tumors typically show p53 mutations. Although the two pathways show distinct molecular alterations, there can be some overlap. ${ }^{4,5}$ Some serous carcinomas may arise in endometrioid carcinomas through p53 mutations, resulting in mixed endometrioid and serous carcinomas. The presence of an endometrioid component in such tumors seems to have no impact on the survival. ${ }^{4}$

Endometrial serous carcinomas are usually described as tumors with a prominent papillary architecture, markedly atypical nuclei, and frequent association with psammoma bodies. ${ }^{6}$ Although many uterine serous carcinomas can show these typical features, there are a substantial number that do not; these are composed entirely or predominantly of glands and/or solid areas without a prominent papillary component. ${ }^{1,2}$ Diffuse nuclear atypia, although characteristic of serous carcinoma, is not evident in every case. Similarly, although endometrioid carcinomas are often glandular and may show solid growth and squamous or mucinous differentiation, there are some endometrioid carcinomas with a prominent papillary architecture. ${ }^{7}$ Endometrioid carcinomas can have micropapillae and slit-like glandular spaces, and can sometimes be associated with psammoma bodies. ${ }^{8}$ Moreover, endometrioid carcinomas can show focal severe nuclear atypia, raising the possibility of serous carcinoma. Endometrial carcinomas with serous and endometrioid features that appear to be superimposed or mixed can be difficult to classify by morphologic examination alone., ${ }^{1,2}$ An accurate diagnosis can be particularly difficult on small endometrial biopsies. ${ }^{9,10}$ In the series by Bristow et $a l,{ }^{9}$ serous carcinomas were correctly diagnosed in only $76 \%$ of pre-operative biopsies and three of the six cases not diagnosed as uterine serous carcinoma were classified as low-grade (FIGO grade 1-2) endometrioid carcinomas.

Distinction between uterine serous carcinoma and endometrioid carcinoma is clinically very important. Serous carcinomas of the endometrium are the prototype of type 2 tumors that frequently have extra-uterine disease and pursue an aggressive clinical course. ${ }^{3,9-12}$ Endometrioid carcinomas (type 1 tumors), on the other hand, especially when low grade, are associated with favorable clinical outcomes. Uterine serous carcinoma characteristically metastasize to the peritoneum, even more frequently than other high-grade endometrial carcinomas. ${ }^{13}$ As a result, gynecologic oncologists usually perform extensive, ovarian cancer-type staging surgeries, including omentectomy. ${ }^{14} \mathrm{~A}$ substantial number of uterine serous carcinoma patients might be under- staged and undertreated if this type of staging surgery is not performed..$^{9,15-17}$

Tp53 gene mutations are a common genetic event in cancers ${ }^{18,19}$ and immunohistochemistry has been shown to be an effective means for their detection. ${ }^{20,21}$ Most Tp53 mutations in uterine serous carcinomas are missense, which results in an altered p53 protein that can be shown by immunohistochemistry because of a prolonged half life. ${ }^{22}$ Variable amounts and staining intensity (from any staining to $50 \%$ ) for p53 have been interpreted as evidence of p53 overexpression in the published literature, ${ }^{16,23-25}$ but the presence of strong nuclear staining in $>75 \%$ of tumor cells has been shown, more specifically, to correlate with the presence of Tp53 mutations. ${ }^{26}$ However, complete absence of p53 by immunohistochemistry may also be indicative of Tp53 mutations, as nonsense or frameshift mutations in p53 can result in a protein that is undetectable by immunohistochemistry. ${ }^{26,27}$

Overexpression of p53 by immunohistochemistry is seen in the vast majority of uterine serous carcinomas. ${ }^{24,28-33}$ Tp53 mutations can be seen in intraepithelial carcinomas and are thought to occur early in the pathogenesis of uterine serous carcinoma. ${ }^{26}$ Among endometrioid carcinomas, p53 overexpression has been reported in a minority of cases, mainly tumors classified as FIGO grade 3 endometrioid carcinomas. ${ }^{31-33}$ Mixed endometrioid and serous carcinomas often show p53 overexpression. ${ }^{30}$ Most studies that address p53 in endometrial carcinomas focus on the association between p53 overexpression and serous histology. ${ }^{24,28-30}$ The data regarding the prognostic significance of p53 overexpression in endometrial carcinomas, however, is controversial. Some studies have shown p53 status to be independently predictive of clinical outcome, ${ }^{16,24,25,34-37}$ whereas in other studies, p53 failed to retain prognostic significance on multivariate analysis. ${ }^{38,39}$ These studies were performed on well-defined and typical subsets of endometrial carcinomas.

This study, in contrast, examines morphologically ambiguous endometrial carcinomas without compelling serous or endometrioid features. This group of tumors is particularly relevant for study as it approximates the types of cases frequently subjected to p53 immunohistochemistry in clinical practice. These endometrial carcinomas frequently exhibit low-grade or glandular architecture with at least focal high-grade nuclei. The primary aim of our study was to assess the clinical significance of p53 overexpression in such morphologically ambiguous endometrial carcinomas. Related aims were (1) to assess interobserver diagnostic concordance for histologic subclassification of these difficult to classify endometrial tumors using a panel of pathologists with and without gynecologic pathology expertise and (2) to elucidate histologic features found in these tumors that correlate with p53 status. 


\section{Materials and methods}

The surgical pathology database from August 2002 to August 2007 was searched after IRB approval to identify all endometrial carcinomas on which a p53 stain had been obtained. We included only those cases for study in which the p53 stain was ordered by a pathologist from our institution. All available H\&E slides were re-reviewed by two gynecologic pathologists to exclude all obvious cases of serous or endometrioid carcinoma. The remaining cases were termed as 'morphologically ambiguous carcinomas' for the purposes of this study. In practice, we routinely classify endometrial tumors on $\mathrm{H} \& \mathrm{E}$ examination alone. Immunohistochemistry, such as the p53 stain, is reserved for cases in which the diagnosis is uncertain or debatable. The usual differential diagnosis in such cases is serous carcinoma versus endometrioid carcinoma FIGO grade 2 (specifically those with glandular or lowgrade architecture and high-grade nuclei), but other subtypes, particularly clear cell carcinoma, are occasionally considered.

The immunohistochemical staining method for p53 remained the same throughout the study period. Briefly, antigen retrieval was performed using citrate $\mathrm{pH} 6.00$ using heat (microwave oven) at $100^{\circ} \mathrm{C}$ for 4 min. Four-micrometer sections of formalin-fixed, paraffin-embedded sections were stained with monoclonal p53 antibody (DO-7, Dako, pre-diluted). Antibody-antigen complexes were detected using DAB I-view detection kit (Ventana, Tucson, AZ) following the manufacturer's instructions.

An adaptation of the German immunoreactive score was used for evaluation of immunohistochemistry. ${ }^{40}$ This score assigns subscores for intensity of immunoreactivity $(0-3+)$ and its distribution $(0-4+)$. These scores were then multiplied to yield a score from 0 to 12 . For p53, only tumors with an immunoreactive score of 9 or 12 was interpreted as evidence of p53 overexpression, as a score of 9 or more (strong nuclear staining in $80 \%$ tumor cells) has been shown to correlate with the presence of Tp53 mutations ${ }^{26}$ (Figure 1). A tumor with a final score of 1-8 was classified as "no p53 overexpression' (Figure 2). Tumors with complete absence of p53 staining were defined as 'p53 null' (Figure 3) and were included within the group with no p53 overexpression for the purpose of this study.

All available $\mathrm{H} \& \mathrm{E}$ slides were reviewed by two pathologists with subspecialty training or experience in gynecologic pathology (RAS and KG). All cases that were considered morphologically diagnostic of serous or endometrioid carcinoma were excluded from the study. The slides were also reviewed by three more pathologists (two board eligible surgical pathology fellows and 1 attending pathologist) without expertise in gynecologic pathology. Morphologic assessment was performed without knowledge of the diagnosis of record, the p53 status or clinical outcome. Cases were coded as

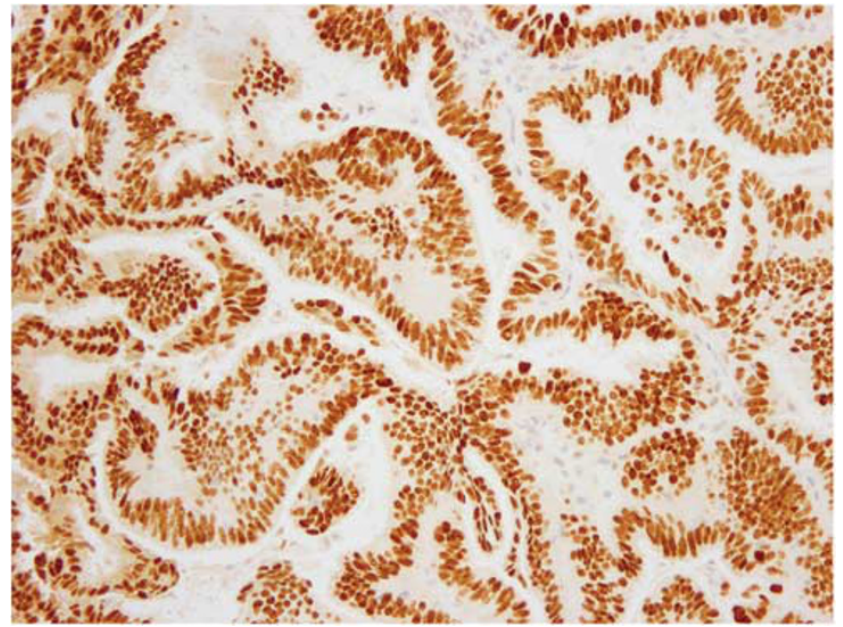

Figure 1 Only diffuse, strong nuclear staining for p53 in $>75 \%$ of tumor cells (immunoreactive score $\geq 9$ ) was interpreted as p53 overexpression.

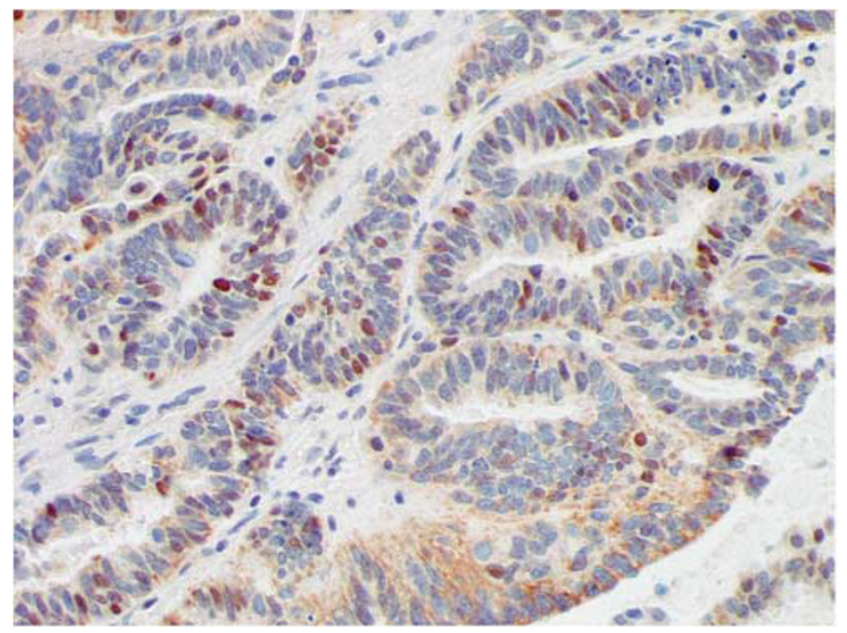

Figure 2 Patchy p53 staining (immunoreactive score 1-6) was not interpreted as p53 overexpression.

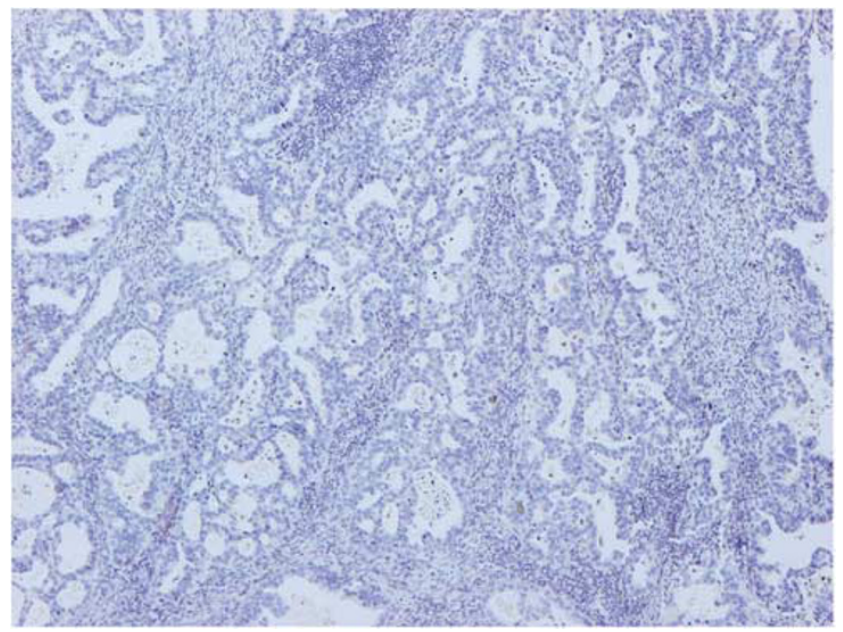

Figure 3 Complete absence of p53 staining (p53 null) may be consistent with serous differentiation and should be distinguished from patchy p53 staining. 
'favor endometrioid', 'favor serous' or 'other'. Nuclei were classified as high grade when they showed at least threefold variation in size and/or shape and displayed prominent nucleoli. Diffuse high nuclear grade was defined as the presence of high-grade nuclei in $>75 \%$ of the tumor cells.

The interobserver concordance between the two gynecologic reviewers was assessed, and their consensus diagnosis was compared with that of the three non-specialized pathologists. The slides were then re-reviewed with knowledge of the p53 status to assess for histologic features that correlated with p53 expression patterns. Clinical data were abstracted from the electronic medical records.

The relationships between clinicopathologic variables and progression-free survival and disease-specific survival were examined using the log-rank test. The probability of freedom from disease progression and death because of disease were estimated using the Kaplan-Meier method. The effect of p53 immunohistochemistry status on the clinical outcomes adjusting for variable, whose $P$-value was $<0.05$ in univariate analysis, was assessed using bivariate stratified exact log-rank test. Interobserver diagnostic concordance was measured using kappa statistics. The relationships between continuous or categorical clinicopathologic variables and p53 status were examined using Wilcoxon-rank-sum test, $\div^{2}$ or Fisher's exact test. All statistical analyses were performed using SAS (SAS Institution) or StatXact software (Cytel Software Corporation). A two-sided $P$-value $(<0.05)$ was considered as significant.

\section{Results}

\section{Patient and Tumor Characteristics}

We identified 40 endometrial carcinomas on which a p53 stain had been ordered by an MSKCC pathologist. Five cases were excluded from the study on review by the gynecologic pathologists, as they were thought to show histologic features diagnostic of serous or endometrioid carcinoma. The remaining 35 morphologically ambiguous endometrial carcinomas formed our study group. Table 1 describes the characteristics of our cohort. The median patient age was 66 years (range 33-79 years). Most patients (83\%) were Caucasian and many had high body mass index (BMI $\geq 25)(63 \%)$. A p53 stain was performed on the hysterectomy specimen in 33 of 35 cases. Of these, p53 stain was also performed on the preceding biopsy in three cases. A p53 stain was performed only on the biopsy in two cases. One did not have residual carcinoma in the hysterectomy specimen, whereas in the other case, no material was available to perform the p53 stain on the hysterectomy specimen. In 14 of the 30 cases in which p53 was performed on the hysterectomy specimen, the possibility of a serous
Table 1 Patient and tumor characteristics by p53 immunohistochemistry status

\begin{tabular}{|c|c|c|c|}
\hline Variable & $\begin{array}{l}\text { All MACs } \\
(\mathrm{n}=35)\end{array}$ & $\begin{array}{c}\text { p53 } \\
\text { overexpression } \\
(\mathrm{n}=17)\end{array}$ & $\begin{array}{c}\text { No p53 } \\
\text { overexpression } \\
\quad(\mathrm{n}=18)\end{array}$ \\
\hline $\begin{array}{l}\text { Median age, } \\
\text { years (range) }\end{array}$ & $66(33-79)$ & $68(47-77)$ & $57(33-79)$ \\
\hline \multicolumn{4}{|l|}{ Race } \\
\hline Caucasian & $29(83)$ & $14(82)$ & $15(83)$ \\
\hline Other & $4(11)$ & 3 (18) & $1(6)$ \\
\hline NA & $2(6)$ & $0(0)$ & $2(11)$ \\
\hline \multicolumn{4}{|l|}{$B M I$} \\
\hline Normal & $11(31)$ & $4(24)$ & $7((39)$ \\
\hline $\begin{array}{l}\text { Overweight/ } \\
\text { obese (BMI } \geq 25 \text { ) }\end{array}$ & $22(63)$ & $12(71)$ & $10((56)$ \\
\hline NA & $2(6)$ & $1(6)$ & $1(6)$ \\
\hline \multicolumn{4}{|l|}{ FIGO stage (1988) } \\
\hline I & $18(51)$ & $8(47)$ & $10(56)$ \\
\hline III & $13(37)$ & $6(35)$ & 7 (39) \\
\hline IV & $2(6)$ & $2(12)$ & $0(0)$ \\
\hline Unstaged & $1(3)$ & $1(6)$ & $1(6)$ \\
\hline \multicolumn{4}{|c|}{ Lymphovascular invasion } \\
\hline Absent & $20(57)$ & $9(53)$ & $11(61)$ \\
\hline Present & $15(43)$ & $8(47)$ & 7 (39) \\
\hline \multicolumn{4}{|c|}{ Dissemination at surgery } \\
\hline None & $21(60)$ & $9(53)$ & $12(67)$ \\
\hline Lymphatic & $6(17)$ & $3(18)$ & $3(17)$ \\
\hline Peritoneal & $8(23)$ & $5(29)$ & 3 (17) \\
\hline
\end{tabular}

Data presented as $n(\%)$.

MAC, morphologically ambiguous endometrial carcinoma; NA, not available; BMI, body mass index.

component or 'high-grade' component was raised on the preceding biopsy specimen.

In patients who were surgically staged $(n=33)$, approximately half presented at early stage (FIGO stage I-II; 51\%), whereas the remainder present at an advanced stage (FIGO stage III-IV; 49\%). Of the 33 patients who were surgically staged, 20 underwent omentectomy and lymph node dissection, whereas the remainder underwent lymph node dissection without omentectomy. Seventeen patients received chemo-radiation, nine received chemotherapy alone, five received radiation alone, whereas four patients received no adjuvant treatment.

\section{Morphologic Features}

The endometrial carcinomas consisted of a heterogeneous group of tumors with variable morphologic features and were defined by the absence of characteristics that permitted confident histologic subclassification without the use of immunohistochemistry. In general, the differential diagnosis included FIGO grade 2 endometrioid carcinoma (those with low-grade or glandular architecture 
and high-grade nuclei) and uterine serous carcinoma. The endometrial carcinomas in our study included tumors that showed overlapping histologic features of endometrioid and serous carcinoma throughout as well as those that seemed to show different components with serous and endometrioid features. Seven tumors $(20 \%)$ were associated with an endometrial polyp. In tumors with adjacent endometrium present for evaluation, hyperplasia was seen in 17 cases, atrophic endometrium was noted in 8 cases, whereas 2 cases showed areas of both atrophy and hyperplasia. All the tumors showed at least focal glandular architecture. A solid growth pattern was noted in a minority of cases $(n=9,25 \%)$ and usually accounted for $<50 \%$ of the tumor volume. Papillary architecture, either focal to predominant, was present in 28 of 35 cases (80\%), whereas psammoma bodies were only infrequently seen (two cases, $5 \%$ ). Foci of squamous or mucinous differentiation were observed in 12 cases (34\%). Twelve of the 35 cases displayed diffuse high nuclear grade $(35 \%)$. All remaining cases showed at least focal high-grade nuclei present in $10-50 \%$ of tumor.

The morphologic spectrum ranged from glandular tumors with high nuclear grade (architectural-cytologic dysynchrony) (Figure 4) to others that were predominantly papillary, but lacked significant nuclear atypia. Some gland-forming tumors were apparent in a background of atrophy. There were several tumors that had a more endometrioid appearance, but showed surface papillary growth and high nuclear grade, whereas others had intraglandular slit-like spaces and micropapillary architecture, leading to diagnostic confusion.

Two recognizably different components were observed in 6 of the 35 cases, although they all

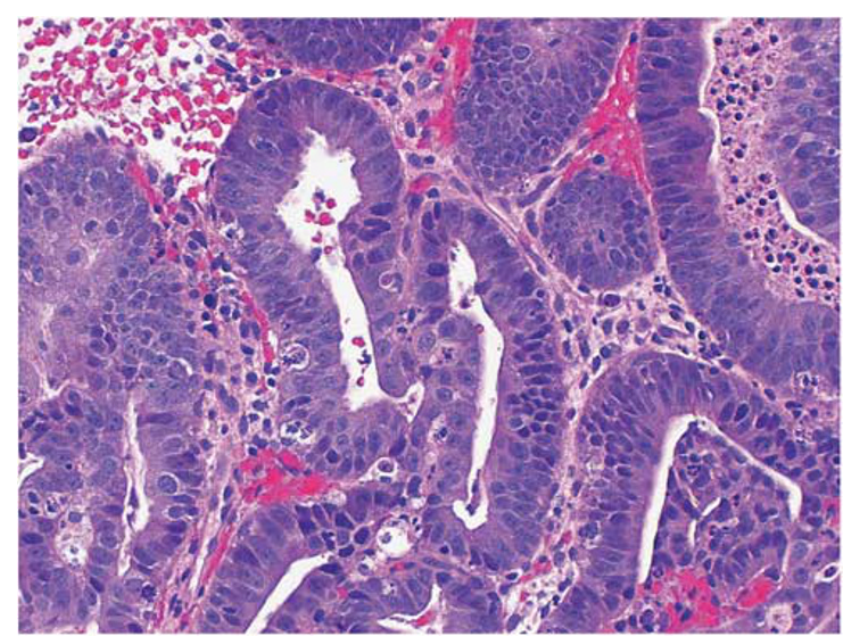

Figure 4 The presence of architectural-cytologic dysynchrony as seen in this tumor (glandular architecture with high-grade nuclei) was often associated with p53 overexpression and aggressive clinical behavior. showed large morphologically ambiguous areas as well. These tumors showed morphologic heterogeneity, characterized by endometrioid-like foci with hyperplasia and/or squamous or mucinous metaplasia, whereas other areas showed glandular and/or papillary architecture with slit-like compressed lumina and very high nuclear grade, features suggestive of serous carcinoma.

\section{Immunohistochemical Staining Results}

Immunohistochemistry for p53 was analyzed in all cases and 17 of $35(48 \%)$ showed p53 overexpression (IHC score $\geq 9$ ). Among the 18 cases that did not show p53 overexpression, 2 cases showed no staining at all for p53 (score of 0; 'p53 null'), whereas the remainder showed patchy staining (immunoreactive score of 1-6). The clinicopathologic factors of patients on the basis of the p53 status are listed in Table 1. Although the patients with p53 overexpression were older, more frequently showed peritoneal dissemination at surgery and presented at higher stages compared with those without p53 overexpression, none of these differences was statistically significant ( $P$-values $0.36-0.74$ ).

\section{Tumor Morphology and Correlation with p53 Status}

Diagnostic agreement was reached in the majority of cases (29 of $35,83 \%$, Table 2) when the cases were reviewed by two gynecologic pathologists (kappa $=0.70$; substantial agreement). There was still diagnostic discordance in six cases. The differential diagnosis in most cases (34 of 35) included uterine serous versus endometrioid carcinoma, whereas in the remaining case, clear cell versus serous carcinoma were the diagnostic considerations.

When applying the morphologic diagnosis rendered by the gynecologic pathologists, p53 overexpression was highly associated with 'favor serous' morphology $(P=0.005)$ (Table 2). In 13 patients in which the two pathologists agreed with the morphologic impression of 'favor serous,' 10 (77\%) showed p53 overexpression, whereas only 4 of the $16(25 \%)$ 'favor endometrioid' cases had overexpression of p53.

The cases were also reviewed by three other pathologists without subspecialty expertise (Table 3). The morphologic concordance rates between the consensus diagnosis of the two specialized pathologists versus each of the other three pathologists showed only poor to fair reproducibility $(\mathrm{kappa}=0.13-0.25)$. There was morphologic agreement between all five pathologists in only six of the 35 cases $(17 \%)$. The correlation between the morphologic diagnosis and p53 status was variable for the non-specialized pathologists, with $41-65 \%$ of cases classified as 'favor serous' showing overexpression of p53. As many as 10 of the 17 cases 
Table 2 Gynecologic pathology review diagnoses and correlation with p53 immunohistochemistry status

\begin{tabular}{|c|c|c|c|c|c|c|}
\hline \multirow[t]{2}{*}{$\begin{array}{l}\text { Gynecologic } \\
\text { pathologic diagnosis }\end{array}$} & \multirow[t]{2}{*}{$\begin{array}{c}S S P \# 1 \\
(\mathrm{n}=35)^{\mathrm{a}}\end{array}$} & \multirow[t]{2}{*}{$\begin{array}{l}S S P \# 2 \\
(\mathrm{n}=35)\end{array}$} & \multirow[t]{2}{*}{$\begin{array}{l}\text { Consensus } \\
\text { diagnosis }(\mathrm{n}=29)\end{array}$} & \multicolumn{3}{|c|}{$\begin{array}{c}\text { p53 expression in cases with consensus } \\
\text { diagnosis between gynecologic pathologists }(\mathrm{n}=29)\end{array}$} \\
\hline & & & & $\begin{array}{l}\text { p53 overexpression } \\
(\mathrm{n}=14)\end{array}$ & $\begin{array}{l}\text { No p53 overexpression } \\
\text { (n=15) }\end{array}$ & $\mathrm{P}$-value ${ }^{\mathrm{b}}$ \\
\hline Favor endometrioid & $19(54 \%)$ & $18(51 \%)$ & 16 & 4 of $16(25 \%)$ & 12 of $16(75 \%)$ & 0.005 \\
\hline Favor serous & $15(43 \%)$ & $17(49 \%)$ & 13 & 10 of $13(77 \%)$ & 3 of $13(23 \%)$ & \\
\hline Other & $1(3 \%)^{\mathrm{c}}$ & $0(0 \%)$ & & & & \\
\hline Agreement & \multicolumn{3}{|c|}{29 of $35(83 \%)$ cases $(\text { kappa }=0.70)^{\mathrm{d}}$} & & & \\
\hline
\end{tabular}

${ }^{\mathrm{a}}$ SSP, sub-specialized gynecologic pathologist.

${ }^{\mathrm{b}}$ On the basis of $\chi^{2}$ test.

${ }^{\mathrm{C}}$ Favor clear cell carcinoma.

d Analysis was based on 34 cases; one favor clear cell carcinoma was excluded from analysis.

Table 3 Morphologic diagnoses rendered by sub-specialized pathologists (SSP) and non-specialized pathologists (NSP) for morphologically ambiguous endometrial carcinomas with p53 immunohistochemistry status ${ }^{\mathrm{a}}$

\begin{tabular}{|c|c|c|c|c|c|c|}
\hline p53 status & Morphology diagnosis & $S S P \# 1$ & $S S P \# 2$ & NSP \# 1 & NSP \# 2 & NSP \# 3 \\
\hline \multirow[t]{3}{*}{ p53 overexpression $(n=17)$} & Favor serous & $10(59)$ & $13(76)$ & $8(47)$ & $11(65)$ & $7(41)$ \\
\hline & Favor endometrioid & $6(35)$ & $4(24)$ & $8(47)$ & $3(18)$ & $10(59)$ \\
\hline & Other & $1(6)$ & $0(0)$ & $1(6)$ & $3(18)$ & $0(0)$ \\
\hline \multirow[t]{3}{*}{ No p53 overexpression $(n=18)$} & Favor serous & $5(28)$ & $4(22)$ & $5(28)$ & $9(50)$ & $5(28)$ \\
\hline & Favor endometrioid & $13(72)$ & $14(78)$ & $11(61)$ & $9(50)$ & $13(72)$ \\
\hline & Other & $0(0)$ & 0 & $2(11)$ & $0(0)$ & $0(0)$ \\
\hline
\end{tabular}

Data presented as $n(\%)$.

${ }^{\mathrm{a}}$ The morphologic concordance between consensus diagnosis of the two SSP and each of the three NSP, kappa value was of $0.13-0.25$.

$(58 \%)$ with p53 overexpression were diagnosed as 'favor endometrioid' by the non-gynecologic pathologists (Table 3).

These analyses were also performed after excluding the six mixed cases (data not shown) and there was no significant difference in the diagnostic concordance results.

\section{Clinical Outcome}

The median follow-up was 2.8 years for survivors (range, 0.5-6.5 years). Two patients were lost to follow up after surgery and were excluded from the analyses. Nine patients developed disease progression during follow-up. The median progression-free survival was 6.1 years (95\% CI not reached). Univariate progression-free survival analysis showed that overexpression of p53 (immunoreactive score of $\geq 9$ versus $<9$ ) was significantly associated with inferior progression-free survival $(P=0.02)$ (Table 4) (Figures 5a and b). The 3-year progression-free survival was $94 \%$ in patients with no p53 overexpression compared with $52 \%$ in patients with p53 overexpression. None of the other factors including age, stage, BMI, lymphovascular invasion or consensus diagnosis was significantly associated with progression-free survival.

Six patients died of disease during follow-up and one patient died of other causes. Univariate analysis revealed that high stage (stage III-IV), favor serous morphology on review, p53 overexpression and presence of lymphovascular invasion were associated with inferior disease-specific survival (disease-specific survival, $P=0.02,0.002,0.003$, 0.03 , respectively), but age and BMI were not (Table 4) (Figures 6a and b). Additional bivariate stratified analysis showed that p53 overexpression remained significantly associated with a shorter disease-specific survival after adjusting for variables whose $P$-values were $<0.05$ on univariate analysis, including stage and lymphovascular invasion $(P=0.009$ and 0.006 , respectively, by exact log-rank test). As the p53 status and gynecologic pathology review diagnosis were strongly correlated, we were unable to accurately discriminate the confounding effects in a bivariate setting.

There were a total of 13 cases with discrepancy between the gynecologic pathologists' consensus diagnosis and p53 status or diagnostic discordance between the two pathologists. Of these, seven tumors showed p53 overexpression, whereas six 
Table 4 Univariate analysis of progression-free survival (PFS) and disease-specific survival (DSS)

\begin{tabular}{|c|c|c|c|c|c|c|}
\hline \multirow[b]{2}{*}{ Variable } & \multicolumn{3}{|c|}{ PFS } & \multicolumn{3}{|c|}{ DSS } \\
\hline & $C /$ total $N^{\mathrm{a}}$ & 3-year PFS $(95 \% C I)^{\mathrm{b}}$ & P-value & $C /$ total $N^{\mathrm{a}}$ & 3-year DSS $(95 \% C I)^{\mathrm{b}}$ & $\mathrm{P}$-value ${ }^{\mathrm{b}}$ \\
\hline \multicolumn{7}{|l|}{ Age (years) } \\
\hline$<65$ & $13 / 16$ & $80 \%(51-93 \%)$ & 0.48 & $15 / 16$ & $93 \%(61-99 \%)$ & 0.13 \\
\hline$\geq 65$ & $11 / 17$ & $67 \%(37-85 \%)$ & & $12 / 17$ & $68 \%(34-87 \%)$ & \\
\hline \multicolumn{7}{|l|}{ FIGO stage (1988) } \\
\hline I & $14 / 17$ & $88 \%(61-97 \%)$ & 0.18 & $17 / 17$ & $100 \%(100-100 \%)$ & 0.02 \\
\hline III/IV & $10 / 15$ & $63 \%(32-83 \%)$ & & $10 / 15$ & $68 \%(33-87 \%)$ & \\
\hline \multicolumn{7}{|l|}{$B M I$} \\
\hline Normal & $6 / 11$ & $64 \%(30-85 \%)$ & 0.45 & $8 / 11$ & $81 \%(42-95 \%)$ & 0.98 \\
\hline Overweight/obese $(\mathrm{BMI} \geq 25)$ & $17 / 21$ & $80 \%(55-92 \%)$ & & $18 / 21$ & $79 \%(43-93 \%)$ & \\
\hline \multicolumn{7}{|l|}{ Lymphovascular invasion } \\
\hline Absent & $15 / 19$ & $84 \%(57-94 \%)$ & 0.21 & $18 / 19$ & $94 \%(67-99 \%)$ & 0.03 \\
\hline Present & $9 / 14$ & $59 \%(27-81 \%)$ & & $9 / 14$ & $61 \%(24-84 \%)$ & \\
\hline \multicolumn{7}{|l|}{ Gynecologic pathology } \\
\hline \multicolumn{7}{|l|}{ Review diagnosis } \\
\hline Favor endometrioid & $13 / 15$ & $87 \%(56-96 \%)$ & 0.11 & $15 / 15$ & $100 \%(100-100 \%)$ & 0.002 \\
\hline Favor serous & $6 / 12$ & $56 \%(24-79 \%)$ & & $7 / 12$ & $50 \%(13-79 \%)$ & \\
\hline \multicolumn{7}{|l|}{ p53 } \\
\hline No overexpression & $16 / 17$ & $94 \%(65-99 \%)$ & 0.02 & $17 / 17$ & $100 \%(100-100 \%)$ & 0.003 \\
\hline Overexpression & $8 / 16$ & $52 \%(25-74 \%)$ & & $10 / 16$ & $54 \%(19-79 \%)$ & \\
\hline
\end{tabular}

${ }^{\mathrm{a}} \mathrm{C}$, censored; two patients lost to follow up after surgery; 33 patients (maximum) were included in the analyses.

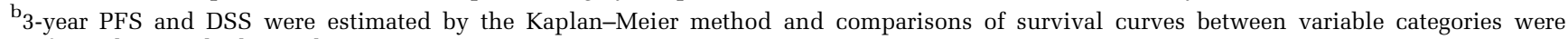
performed using the log-rank test.

did not. Of note, four of the seven patients whose tumors showed p53 overexpression presented at advanced stages (III-IV), and are either alive with disease or dead of disease.

These analyses were also performed after excluding the six mixed cases (data not shown) and p53 overexpression remained significantly associated with survival on univariate analysis regardless of whether mixed cases were included in the data set.

Some examples of morphologically ambiguous carcinomas with their p53 status and clinical outcomes are illustrated in Figures 7-9.

\section{Correlation of Morphologic Features with p53 Status}

The tumors were re-reviewed to assess for specific morphologic features that could potentially correlate with the p53 expression pattern (Table 5). The analyses were performed including the mixed cases and also after excluding them. Both approaches revealed that the presence of diffuse marked nuclear atypia ( $P=0.0002$ and 0.0005 , respectively) was the only feature that was significantly associated with the presence of p53 overexpression. Although serous intraepithelial carcinoma (Figure 10) and slit such as compressed spaces (Figure 11) were seen more often in cases with p53 overexpression, they were not statistically significant in either analyses. No other features were significantly associated with p53 overexpression. Psammoma bodies were observed in only two cases and both lacked p53 overexpression. Myometrial invasion in the form of single 'gaping glands' was seen only in two cases, both with p53 overexpression (Figure 12).

\section{Discussion}

Our study shows that application of p53 immunohistochemistry in a subset of morphologically ambiguous endometrial carcinomas is prognostically informative. The clinical outcomes, including progression-free and disease-specific survival, were distinctly different in tumors with p53 overexpression compared with those without p53 overexpression. Six patients died of disease in the former group, whereas none died in the group that lacked p53 overexpression. The clinical course of the morphologically ambiguous endometrial carcinomas with p53 overexpression more closely resembled that of typical uterine serous carcinoma, rather than an endometrioid carcinoma. The 3-year progression-free survival was $52 \%$ in tumors with p53 overexpression, compared with $46-54 \%$ for uterine serous carcinomas. ${ }^{14,41}$ Similarly, the 3-year disease-specific survival of morphologically ambiguous endometrial carcinomas with p53 overexpression $(54 \%)$ was similar to uterine serous carcinomas (43-62\%). ${ }^{10,14,41}$ Although multivariate analysis was 

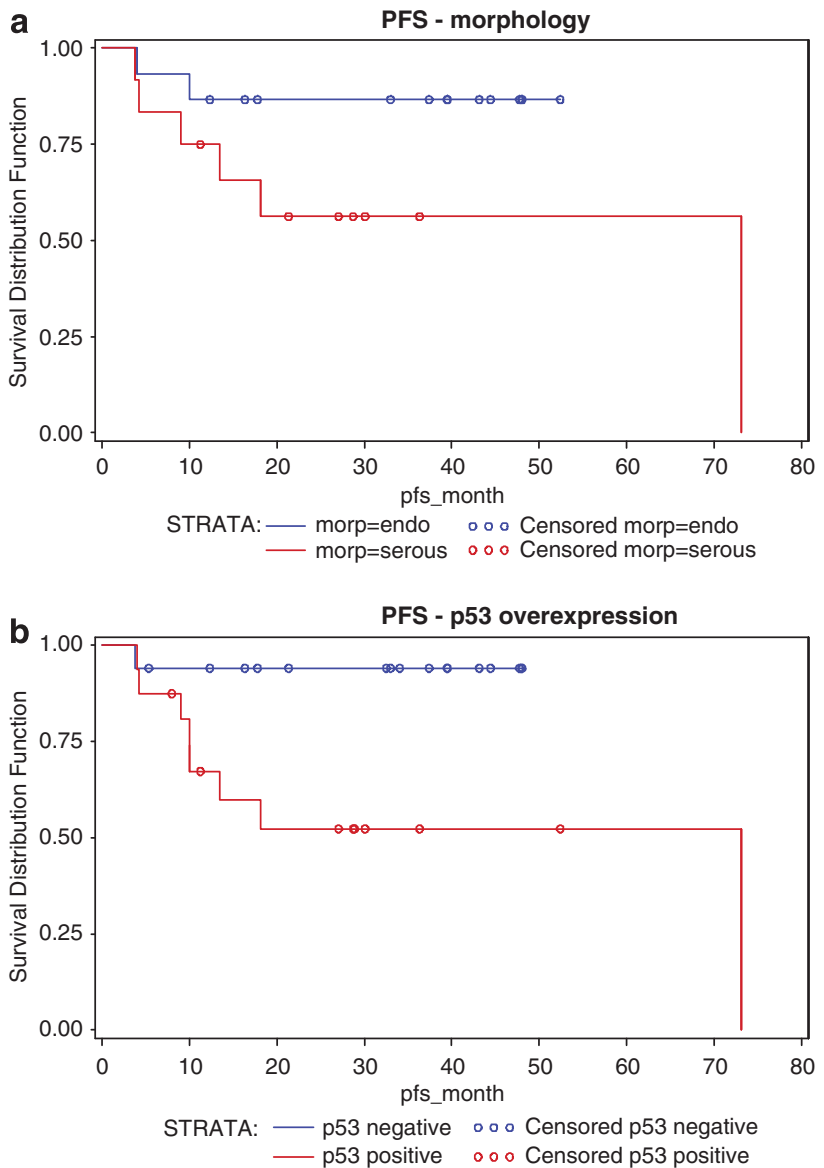

Figure 5 (a) Kaplan-Meier plot of progression-free survival stratified by consensus morphology and (b) Kaplan-Meier plot of PFS stratified by p53 status (morp, morphology; PFS, progression-free survival).

not feasible because of the small number of cases, bivariate stratified analysis showed that p53 status remained significant after adjusting for other significant factors in univariate analysis (stage and lymphovascular invasion).

Morphologically ambiguous endometrial carcinomas with p53 overexpression showed more frequent peritoneal involvement compared with those without p53 overexpression, although this difference was not statistically significant. One of the characteristic features of uterine serous carcinomas is the propensity for peritoneal spread $(60 \%)$, which is much greater even when compared with FIGO grade 3 endometrioid and clear cell carcinomas $(10 \%){ }^{13}$

In our experience, problematic endometrial carcinomas frequently show well-differentiated architectural patterns, coupled with at least focal significant nuclear atypia. All morphologically ambiguous endometrial carcinomas in our study were composed predominantly of glands with variable amounts of papillary growth. Using the FIGO grading system for endometrial endometrioid carcinomas, a tumor composed predominantly of glands

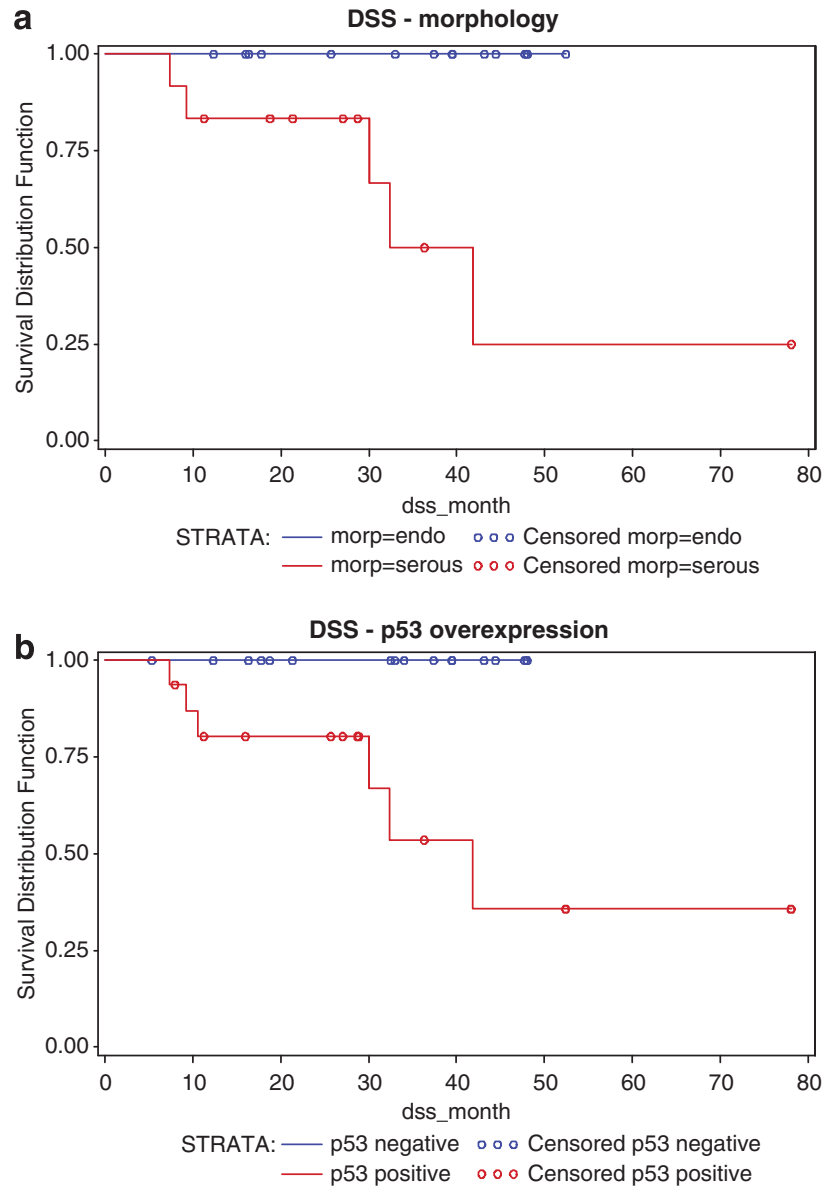

Figure 6 (a) Kaplan-Meier plot of disease-specific survival stratified by consensus morphology and (b) Kaplan-Meier plot of disease-specific survival stratified by p53 status (DSS, diseasespecific survival; morp, morphology).

or papillae (architecture grade 1), even in the presence of high nuclear grade (nuclear grade 3 ), would be classified as FIGO grade 2 endometrioid carcinoma. The differential diagnosis in these challenging cases, therefore, usually involves uterine serous versus FIGO grade 2 endometrioid carcinoma. The 3-year progression-free survival $(94 \%)$ and disease-specific survival (100\%) for morphologically ambiguous endometrial carcinomas lacking p53 overexpression, were similar to that of FIGO grade 2 endometrioid carcinomas $(85 \%))^{42}$

The consensus diagnosis rendered by gynecologic pathologists in this study was as predictive of disease-specific survival as the p53 status, but agreement rates and the ability to predict the p53 status and disease-specific survival for the reviewers without training in gynecologic pathology were comparatively poor. Despite this, it is uncertain whether these results are applicable to other subspecialty reviewers as the two gynecologic pathologists participating in the study are colleagues and have been influenced by each other's diagnostic philosophy. 

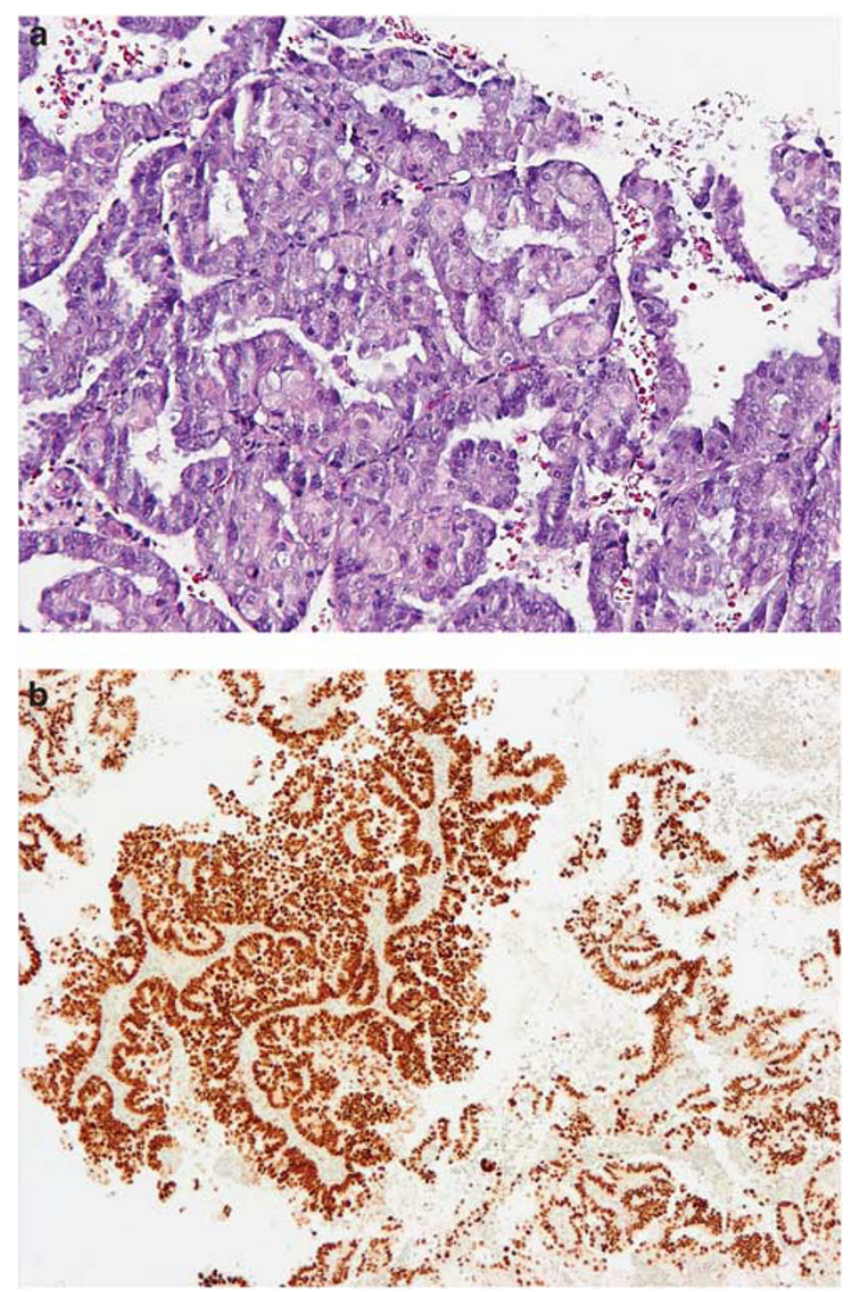

Figure 7 (a and b) P53 staining can be particularly helpful in small, crushed biopsies such as this one. This tumor shows p53 overexpression. The patient presented at stage IIIA and is dead of disease.

A significant number of morphologically ambiguous endometrial carcinomas with p53 overexpression (up to 10 of 17 cases) were classified as favor endometrioid by the non-specialized pathologists. This reflects the diagnostic challenge that these endometrial tumors pose and the potential for undertreatment of such patients. Overall, there was agreement between all five pathologists in only 6 of 35 cases. Therefore, although this distinction can be achieved by H\&E examination by specialized pathologists in many cases, such difficult to diagnose endometrial carcinomas remain a substantial problem for pathologists in general practice.

Our data show that application of p53 immunohistochemistry can serve as an objective prognostic marker. P53 stain is easy to perform and interpret. It should be emphasized that only strong and diffuse p53 staining in $75 \%$ or more of tumor cells ${ }^{26}$ should be accepted as overexpression, although some authors use a $50 \%$ cutoff. ${ }^{23}$ Complete absence of staining for p53 does not exclude serous carcinoma

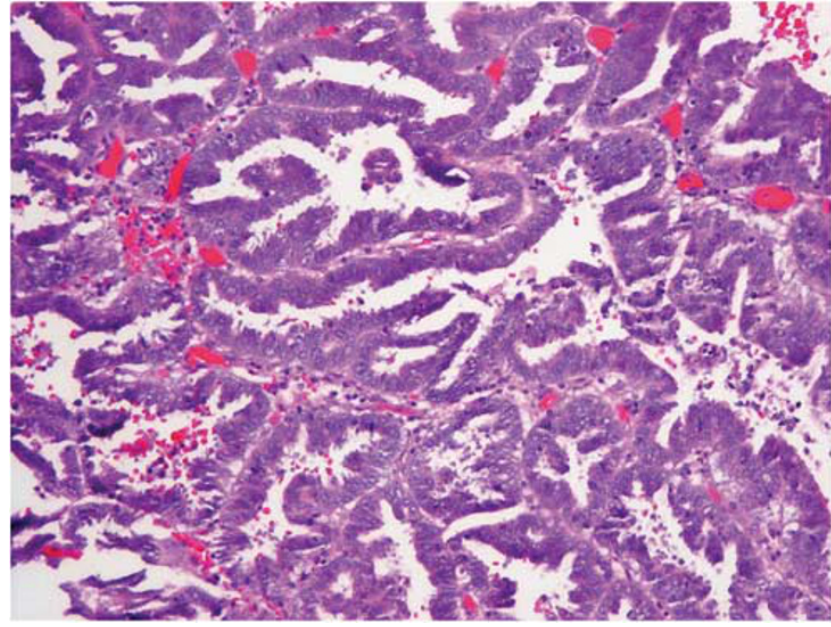

Figure 8 A morphologic consensus diagnosis was not reached in this case. There was no p53 overexpression and the patient has pursued a favorable clinical course.
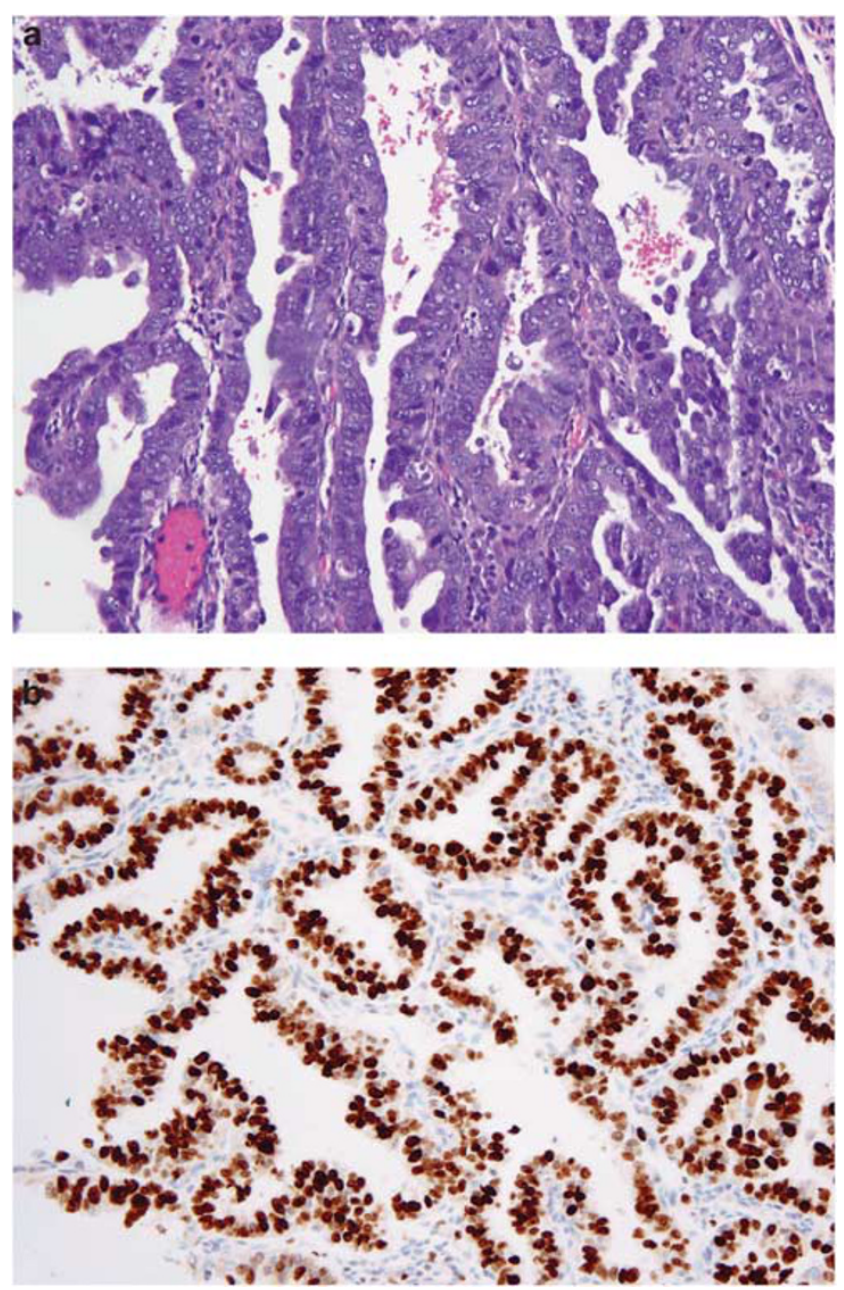

Figure 9 (a and b) The specialized pathologists rendered a diagnosis of 'favor serous', the tumor was p53 null and showed a very high proliferation index by MIB-1 (b). The patient was not staged and lost to follow up. 
Table 5 Relationship between morphologic features and the p53 immunohistochemistry status

\begin{tabular}{|c|c|c|c|}
\hline $\begin{array}{l}\text { Histologic features } \\
\text { ( } n=\text { total number of } \\
\text { cases with each } \\
\text { histologic finding) }\end{array}$ & $\begin{array}{c}\text { No p53 } \\
\text { overexpression } \\
\quad(\mathrm{n}=18)\end{array}$ & $\begin{array}{c}p 53 \\
\text { overexpression } \\
(\mathrm{n}=17)\end{array}$ & P-value ${ }^{\mathrm{a}}$ \\
\hline \multicolumn{4}{|l|}{ Polyp } \\
\hline No & $13(72)$ & $15(88)$ & \multirow[t]{2}{*}{0.40} \\
\hline Yes & $5(28)$ & $2(12)$ & \\
\hline \multicolumn{4}{|l|}{ Hyperplasia ${ }^{\mathrm{b}}$} \\
\hline No & $5(31)$ & $3(27)$ & \multirow{2}{*}{1.0} \\
\hline Yes & $11(69)$ & $8(73)$ & \\
\hline \multicolumn{4}{|l|}{ Atrophy ${ }^{\mathrm{b}}$} \\
\hline No & $11(69)$ & $6(55)$ & \multirow{2}{*}{0.69} \\
\hline Yes & $5(31)$ & $5(45)$ & \\
\hline \multicolumn{4}{|c|}{ Glandular architecture } \\
\hline No & $0(0)$ & $0(0)$ & \multirow[t]{2}{*}{ - } \\
\hline Yes & $18(100)$ & $17(100)$ & \\
\hline \multicolumn{4}{|l|}{ Solid growth } \\
\hline No & $15(83)$ & $11(65)$ & \multirow{2}{*}{0.26} \\
\hline Yes & $3(17)$ & $6(35)$ & \\
\hline \multicolumn{4}{|c|}{ Squamous and/or mucinous metaplasia } \\
\hline No & $10(56)^{x}$ & $13(76)$ & \multirow[t]{2}{*}{0.19} \\
\hline Yes & $8(44)$ & $4(24)$ & \\
\hline \multicolumn{4}{|l|}{$E I C^{\mathrm{b}, \mathrm{c}}$} \\
\hline No & $15(94)$ & $8(73)$ & \multirow[t]{2}{*}{0.27} \\
\hline Yes & $1(6)$ & $3(27)$ & \\
\hline \multicolumn{4}{|l|}{ Papillary architecture } \\
\hline No & $4(22)$ & $3(18)$ & \multirow[t]{2}{*}{1.00} \\
\hline Yes & $14(78)$ & $14(82)$ & \\
\hline \multicolumn{4}{|l|}{ Micropapillae } \\
\hline No & $8(44)$ & $7(41)$ & \multirow[t]{2}{*}{0.85} \\
\hline Yes & $10(56)$ & $10(59)$ & \\
\hline \multicolumn{4}{|c|}{ Slit like compressed spaces } \\
\hline No & $12(67)$ & $8(47)$ & \multirow[t]{2}{*}{0.24} \\
\hline Yes & $6(33)$ & $9(53)$ & \\
\hline \multicolumn{4}{|c|}{ Diffuse marked nuclear atypia } \\
\hline No & $17(94)$ & $6(35)$ & \multirow[t]{2}{*}{0.0002} \\
\hline Yes & $1(6)$ & $11(65)$ & \\
\hline
\end{tabular}

Data presented as $n(\%)$.

${ }^{\mathrm{a}}$ Analysis was on the basis of evaluable cases and $\chi^{2}$ or Fisher's exact test.

${ }^{\mathrm{b}}$ Eight cases (six in p53 overexpression, two in no p53 overexpression) were not evaluable.

${ }^{\mathrm{C}}$ EIC, endometrial intraepithelial carcinoma.

or the presence of Tp53 mutation, however. ${ }^{26}$ The function of p53 immunohistochemistry in endometrial carcinomas has been controversial in no small part because of philosophical differences related to nosology. We do not necessarily advocate the use of p53 staining in such cases as a diagnostic tool, but suggest, instead, that p53 be used as a marker of clinical behavior in diagnostically problematic cases.

Papillary growth, focal high nuclear grade, presence of psammoma bodies, slit such as spaces and

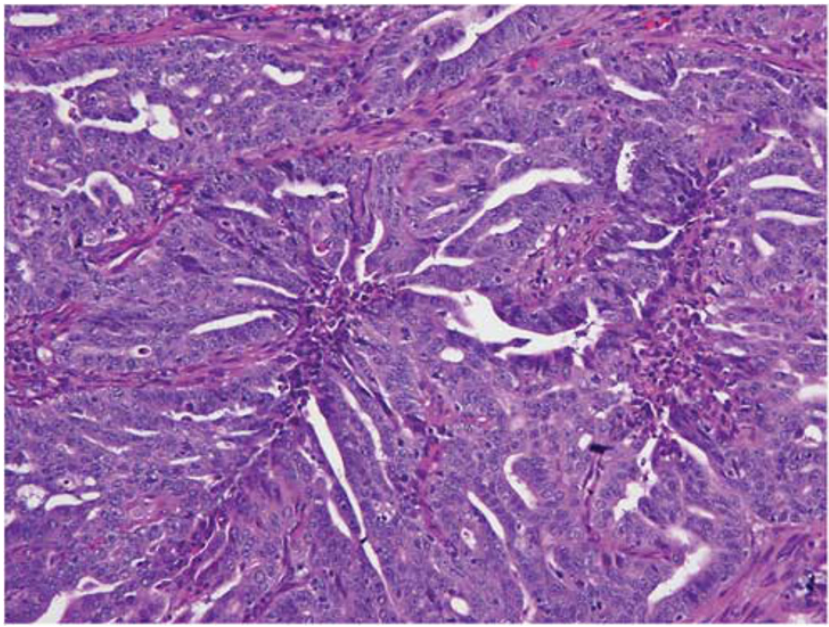

Figure 10 Slit-like spaces were seen more often in tumors with p53 overexpression, but were not specific.

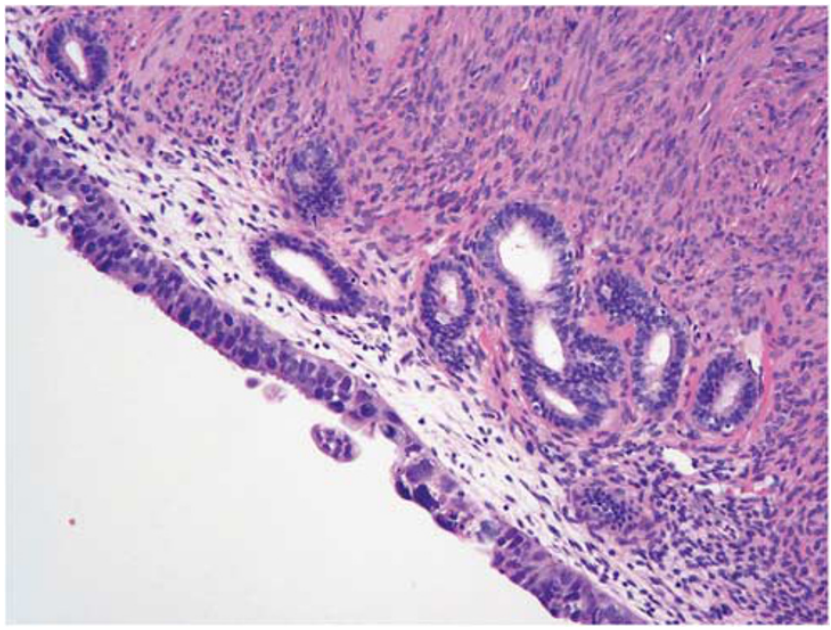

Figure 11 The presence of intraepithelial carcinoma was associated with p53 overexpression.

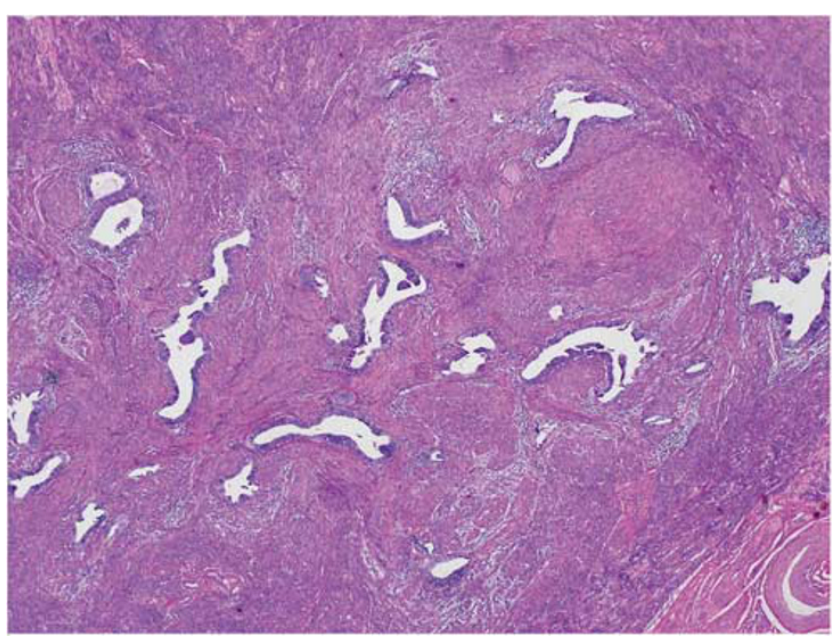

Figure 12 Myometrial invasion in the form of gaping glands was seen only in two cases, both with p53 overexpression. 
micropapillary architecture, all generally thought to be typical of uterine serous carcinoma, were not necessarily found to correlate with p53 overexpression. Instead, the histologic feature that correlated most closely with p53 overexpression was the presence of diffuse marked nuclear atypia. The presence of intraepithelial carcinoma, adjacent atrophic endometrium and architectural-cytologic dyssynchrony (glandular tumor with high nuclear grade) can also be helpful. Conversely, the presence of endometrial hyperplasia and squamous or mucinous differentiation was more frequently observed in tumors that lacked p53 overexpression. However, none of these features was specific and there was a substantial degree of overlap, particularly in mixed tumors. These overlapping histologic features in mixed carcinomas can lead to problems in classification. Of the six mixed cases in our study, there was diagnostic discordance between the two gynecologic pathologists in one case, whereas another case was classified as 'favor endometrioid' by both pathologists. Diagnostic agreement was not reached in any of these six cases by all five pathologists. This reflects the diagnostic challenge that mixed tumors can present, which is the reason we included such cases in our study. Moreover, when the data was analyzed (including clinical outcomes and kappa statistics) after excluding the mixed cases, there was no impact on the results.

If immunohistochemistry for p53 is used, tumors with 'favor serous' morphology and p53 overexpression are diagnosed as serous carcinoma. Likewise, endometrial carcinomas with 'favor endometrioid' features that lack p53 overexpression are classified as endometrioid carcinoma. However, there are still endometrial carcinomas that remain elusive even after careful morphologic and immunohistochemical analysis.

Morphologically ambiguous endometrial carcinomas with p53 overexpression and endometrioid morphologic features or those without a consensus diagnosis may represent serous carcinomas with misleading histologic features, endometrioid carcinomas with p53 mutations, mixed tumors with endometrioid and serous components or unusual endometrial carcinoma variants. Depending on one's preference, endometrial carcinomas, such as these, can be diagnosed as 'high-grade endometrial carcinoma' with a note reporting the presence of p53 overexpression and the potential for aggressive clinical behavior and peritoneal dissemination. It is inappropriate to consider these FIGO grade 2 endometrioid carcinomas, as discussed earlier.

Endometrial tumors that lack p53 overexpression (immunoreactive score of 1-8) and display 'favor serous' morphologic characteristics or defy a consensus diagnosis may represent endometrioid carcinomas, oxyphilic clear cell carcinomas or other endometrial carcinoma variants. Our data suggest that tumors with a consensus diagnosis of 'favor serous' should be considered uterine serous carcinomas irrespective of the p53 status, at least until we have data to prove otherwise. On the other hand, endometrial carcinomas without a consensus diagnosis of serous carcinoma that lack p53 overexpression (immunoreactive score of 1-8) should not be considered serous carcinomas.

Additional immunohistochemical markers that have been shown to correlate with endometrial tumor type and prognosis include p16, PTEN and MIB-1, among others. Strong and diffuse staining for p16 has been shown to correlate with serous morphology. ${ }^{43-45}$ Some studies have suggested that p16 is a more robust marker of serous differentiation than p53, ${ }^{43}$ although other studies have reported an expression gradient, with increasing levels of p16 staining from low- to high-grade endometrioid carcinoma, clear cell carcinoma and, finally, serous carcinoma, ${ }^{45}$ suggesting that additional work is necessary to define relevant diagnostic cutoffs. We are aware of only one study that reports an association between p16 expression and aggressive clinical behavior specifically. ${ }^{46}$ Loss of PTEN staining (or presence of PTEN mutation) has been associated with endometrioid histology and favorable clinical outcomes, ${ }^{1,45,47,48}$ but antibodies against PTEN are notoriously difficult to work with. ${ }^{49}$ Some studies have shown that a combination of markers including p53, p16, PTEN and PR may be superior to p53 alone in discriminating uterine serous versus endometrioid carcinoma. ${ }^{1,50}$ High proliferation index by MIB-1 stain has been shown to be of prognostic significance in endometrial carcinomas in some studies, ${ }^{51-53}$ whereas others have shown no prognostic significance of MIB-1 in high-grade carcinomas of the endometrium. ${ }^{54}$ We use MIB-1 in endometrial cancers that show complete absence of staining for p53 (p53 null), a pattern that has been shown to correlate with the presence of frameshift or nonsense p53 mutations. If such tumors show a very high proliferative rate ( $>80 \%$ ), we favor a diagnosis of serous carcinoma in the appropriate clinical and morphologic context. A MIB-1 stain also helps differentiate serous intraepithelial carcinoma from its putative latent precursor or 'p53 signature,' recently described in the endometrium. ${ }^{55,56}$

In summary, there are occasional endometrial carcinomas that have overlapping histologic features of serous and endometrioid carcinoma and defy accurate and reproducible morphologic subclassification. Our study shows that application of p53 immunohistochemistry in this setting has prognostic value. Morphologically ambiguous endometrial carcinomas with p53 overexpression pursued a more aggressive clinical course than carcinomas without p53 overexpression. This study also shows that while specialized gynecologic pathologists show very good diagnostic agreement and can accurately predict the p53 status in most morphologically ambiguous endometrial carcinomas, pathologists lacking expertise in gynecologic pathology were relatively unsuccessful. As every difficult 
endometrial carcinoma cannot be reviewed by a specialist, p53 immunohistochemistry may be helpful as a more objective means for prognostic assessment and treatment planning in these difficult cases, and, depending on one's viewpoint, could also be used as a diagnostic adjunct.

\section{Acknowledgement}

We thank Dr Richard Zaino and Dr Blake Gilks for their contributions to the manuscript.

\section{Disclosure/conflict of interest}

The authors declare no conflict of interest.

\section{References}

1 Darvishian F, Hummer AJ, Thaler HT, et al. Serous endometrial cancers that mimic endometrioid adenocarcinomas: a clinicopathologic and immunohistochemical study of a group of problematic cases. Am J Surg Pathol 2004;28:1568-1578.

2 Lomo L, Nucci MR, Lee KR, et al. Histologic and immunohistochemical decision making in endometrial adenocarcinoma. Mod Pathol 2008;21:937-942.

3 Bokhman JV. Two pathogenetic types of endometrial carcinoma. Gynecol Oncol 1983;15:10-17.

4 Carcangiu ML, Chambers JT. Uterine papillary serous carcinoma: a study of 108 cases with emphasis on the prognostic significance of associated endometrioid carcinoma, absence of invasion, and concomitant ovarian carcinoma. Gynecol Oncol 1992;47:298-305.

5 Sherman ME. Theories of endometrial carcinogenesis: a multidisciplinary approach. Mod Pathol 2000;13: 295-308.

6 Hendrickson M, Ross J, Eifel P, et al. Uterine papillary serous carcinoma: a highly malignant form of endometrial adenocarcinoma. Am J Surg Pathol 1982;6: 93-108.

7 Murray SK, Young RH, Scully RE. Uterine endometrioid carcinoma with small nonvillous papillae: an analysis of 26 cases of a favorable prognosis tumor to be distinguished from serous carcinoma. Int J Surg Pathol 2000;8:279-289.

8 Parkash V, Carcangiu ML. Endometrioid endometrial adenocarcinoma with psammoma bodies. Am J Surg Pathol 1997;21:399-406.

9 Bristow RE, Asrari F, Trimble EL, et al. Extended surgical staging for uterine papillary serous carcinoma: survival outcome of locoregional (stage I-III) disease. Gyn Oncol 2001;81:279-286.

10 Tay EH, Ward BG. The treatment of uterine papillary serous carcinoma (UPSC): are we doing the right thing? Int J Gynecol Cancer 1999;9:463-469.

11 Lee KR, Belinson JL. Recurrence in noninvasive endometrial carcinoma: relationship to uterine papillary serous carcinoma. Am J Surg Pathol 1991;15: 965-973.

12 Sherman ME, Bitterman P, Rosenshein NB, et al. Uterine serous carcinoma: a morphologically diverse neoplasm with unifying clinicopathologic features. Am J Surg Pathol 1992;16:600-610.

13 Soslow RA, Bissonnette JP, Wilton A, et al. Clinicopathologic analysis of 187 high grade endometrial carcinomas of different histologic subtypes: similar outcome belies distinctive biologic differences. Am J Surg Pathol 2007;31:979-987.

14 Slomovitz BM, Burke TW, Eifel PJ, et al. Uterine papillary serous carcinoma (UPSC): a single institution review of 129 cases. Gynecol Oncol 2003;91:463-469.

15 Carcangiu ML, Tan LK, Chambers JT. Stage IA uterine serous carcinoma: a study of 13 cases. Am J Surg Pathol 1997;21:1507-1514.

16 Geisler JP, Geisler HE, Wiemann MC, et al. p53 expression as a prognostic indicator of 5 year survival in endometrial cancer. Gynecol Oncol 1999;74: 468-471.

17 Goff BA, Kato D, Schmidt RA, et al. Uterine papillary serous carcinoma: patterns of metastatic spread. Gynecol Oncol 1994;54:264-268.

18 Bartek J, Bartkova J, Vojtesek B, et al. Aberrant expression of the p53 oncoprotein is a common feature of a wide spectrum of human malignancies. Oncogene 1991;6:1699-1703.

19 Chang K, Ding I, Kern FG, et al. Immunohistochemical analysis of p53 and her-2/neu proteins in human tumors. J Histochem Cytochem 1991;39:1281-1287.

20 Iggo R, Gatter K, Bartek J, et al. Increased expression of mutant forms of p53 oncogene in primary lung cancer. Lancet 1990;335:675-679.

21 Soong R, Robbins PD, Dix BR, et al. Concordance between p53 protein overexpression and gene mutation in a large series of common human carcinomas. Hum Pathol 1996;27:1050-1055.

22 Harris CC, Hollstein M. Clinical implications of the p53 tumor suppressor gene. N Engl J Med 1993;329: 1318-1327.

23 Alkushi A, Lim P, Coldman A, et al. Interpretation of p53 immunoreactivity in endometrial carcinoma: establishing a clinically relevant cut-off level. Int J Gynecol Pathol 2004;23:129-137.

24 Moll UM, Chalas E, Auguste M, et al. Uterine papillary serous carcinoma evolves via a p53-driven pathway. Hum Pathol 1996;27:1295-1300.

25 Salvesen $\mathrm{HB}$, Iversen OE, Akslen LA. Prognostic significance of angiogenesis and ki-67, p53 and p21 expression: a population based endometrial carcinoma study. J Clin Oncol 1999;17:1382-1390.

26 Tashiro $\mathrm{H}$, Isacson $\mathrm{C}$, Levine $\mathrm{R}$, et al. p53 gene mutations are common in uterine serous carcinoma and occur early in their pathogenesis. Am J Pathol 1997;150:177-185.

27 Lax SF, Kendall B, Tashiro H, et al. The frequency of p53, k-ras mutations, and microsatellite instability differs in uterine endometrioid and serous carcinoma, evidence of distinct molecular genetic pathways. Cancer 2000;88:814-824.

28 Bur ME, Perlman C, Edelmann L, et al. p53 expression in neoplasms of the uterine corpus. Am J Clin Pathol 1992;98:81-87.

29 King SA, Adas AA, Livolsi VA, et al. Expression and mutation analysis of the p53 gene in uterine papillary serous carcinoma. Cancer 1995;75:2700-2705.

30 Sherman ME, Bur ME, Kurman RJ. P53 in endometrial cancer and its putative precursors: evidence for diverse pathways of tumorigenesis. Hum Pathol 1995; 26:1268-1274. 
31 Egan JA, Ionescu MC, Eapen E, et al. Differential expression of WT1 and p53 in serous and endometrioid carcinomas of the endometrium. Int J Gynecol Pathol 2004;23:119-122.

32 Halperin R, Zehavi S, Habler L, et al. Comparative immunohistochemical study of endometrioid and serous papillary carcinoma of endometrium. Eur J Gynaecol Oncol 2001;22:122-126.

33 Soslow RA, Shen PU, Chung MH, Isacson C. Distinctive p53 and mdm2 immunohistochemical expression profiles suggest different pathogenetic pathways in poorly differentiated endometrial carcinoma. Int J Gynecol Pathol 1998;17:129-134.

34 Hamel NW, Sebo TJ, Wilson TO, et al. Prognostic value of p53 and proliferating cell nuclear antigen expression in endometrial carcinoma. Gynecol Oncol 1996;62:192-198.

35 Pisani AL, Barbuto DA, Chen D, et al. Her-2/neu, p53 and DNA analyses as prognosticators for survival in endometrial carcinoma. Obstet Gynecol 1995;85: 729-734.

36 Powell B, Soong R, Grieu F, et al. p53 protein overexpression is a prognostic indicator of poor survival in stage 1 endometrial carcinoma. Int J Oncol 1999;14:175-179.

37 Silverman MB, Roche PC, Kho RM. Molecular and cytokinetic pretreatment risk assessment in endometrial carcinoma. Gyn Oncol 2000;77:1-7.

38 Athanassiadou P, Athanassiades P, Grapsa D, et al. The prognostic value of PTEN, p53 and beta-catenin in endometrial carcinoma: a prospective immunocytochemical study. Int J Gynecol Cancer 2007;17:697-704.

39 Lukes AS, Kohler MF, Pieper CF, et al. Multivariable analysis of DNA ploidy, p53 and her-2/neu as prognostic factors in endometrial cancer. Cancer 1994;73:2380-2385.

40 Remmele W, Stegner HE. Recommendations for uniform definition of an immunoreactive score (IRS) for immunohistochemical estrogen receptor detection (ER-ICA) in breast cancer tissue. Pathologe 1987;8: 138-140.

41 Faratian D, Stillie A, Busby-Earle RMC, et al. A review of the pathology and management of uterine papillary serous carcinoma and correlation with outcome. Int J Gynecol Cancer 2006;16:972-978.

42 Zaino RJ, Kurman RJ, Diana KL, et al. The utility of revised International Federation of Gynecology and Obstetrics histologic grading of endometrial adenocarcinoma using a defined nuclear grading system. Cancer 1995;75:81-86.

43 Chiesa-Vottero AG, Malpica A, Deavers MT, et al. Immunohistochemical overexpression of p16 and p53 in uterine serous carcinoma and ovarian high grade serous carcinoma. Int J Gynecol Pathol 2007;26: 328-333.
44 O’Neill CJ, McCluggage WG. P16 expression in the female genital tract and its value in diagnosis. Adv Anat Pathol 2006;13:8-15.

45 Reid-Nicholson $\mathrm{M}$, Iyengar $\mathrm{P}$, Hummer AJ, et al. Immunophenotypic diversity of endometrial adenocarcinomas: implications for differential diagnosis. Mod Pathol 2006;19:1091-1100.

46 Engelson IB, Stefansson I, Akslen LA. Pathologic expression of p53 or p16 in preoperative curettage specimens identified high risk endometrial carcinomas. Am J Obst Gynecol 2006;195:979-986.

47 Alkushi A, Clarke BA, Akbari M, et al. Identification of prognostically relevant and reproducible subsets of endometrial adenocarcinoma based on clustering analysis of immunostaining data. Mod Pathol 2007;20: 1156-1165.

48 Risinger JL, Hayes K, Maxwell GL. PTEN mutation in endometrial cancers is associated with favorable clinical and pathologic characteristics. Clin Cancer Res 1998;4:3005-3010.

49 Pallares J, Bussaglia E, Martinez-Guitarte JL, et al. Immunohistochemical analysis of PTEN in endometrial carcinomas: a tissue microarray study with a comparison of four commercial antibodies in correlation with molecular abnormalities. Mod Pathol 2005;18:7159-7727.

50 Alkushi A, Kobel M, Kalloger SE, et al. High grade endometrial carcinoma: serous and grade 3 endometrioid carcinoma have different immunophenotypes and outcomes. Modern Path 2009;22:206A.

51 Geisler JP, Geisler HE, Miller GA, et al. MIB-1 in endometrial carcinoma: prognostic significance with 5-year follow-up. Gynecol Oncol 1999;75:432-436.

52 Kallakury BV, Ambros RA, Hayner-Buchan AM, et al. Cell proliferation associated proteins in endometrial carcinomas, including papillary serous and endometrioid subtypes. Int J Gynecol Pathol 1998;17: 320-326.

53 Salvesen HB, Iverson OE, Akslen LA. Identification of high risk patients by assessment of nuclear Ki-67 expression in a prospective study of endometrial carcinomas. Clin Cancer Res 1998;4:2779-2785.

54 Alkushi A, Lim P, Aquino-Parsons C, et al. Markers of proliferative activity are predictors of patient outcome for low-grade endometrioid adenocarcinoma but not papillary serous carcinoma of endometrium. Mod Pathol 2002;15:365-371.

55 Jarboe EA, Pizer ES, Miron A, et al. Evidence for a latent precursor (p53 signature) that may precede serous endometrial intraepithelial carcinoma. Mod Pathol 2009;22:345-350 doi: 10.1038.

56 Jia L, Liu Y, X Y, et al. Endometrial glandular dysplasia with frequent p53 mutation: a genetic evidence supporting its precancer nature for endometrial serous carcinoma. Clin Cancer Res 2008;14:2263-2269. 